\title{
INNER LIMIT DERIVATIONS
}

\author{
RICHARD H. HERMAN ${ }^{1}$
}

\begin{abstract}
We give a condition involving the approximating derivations and a faithful state which, in conjunction with the "core condition", guarantees that the derivation gives rise to an automorphism group of the von Neumann algebra coming from the state via the GNS procedure.
\end{abstract}

One of the basic problems in the theory of unbounded derivations is to find conditions such that the derivation or an extension gives rise to an automorphism group of the algebra in question. In the von Neumann algebra setting the following was proposed, [2].

"Let $\mathfrak{T}$ have a cyclic and separating vector $\xi$, and $H$ be a selfadjoint operator giving rise to a densely defined derivation of $\mathfrak{T}$. If $H \xi=0$ and $\mathscr{D}(\delta) \xi$ is a core for $H$, then $\operatorname{ad}\left(e^{i H t}\right)$ takes $\mathfrak{N}$ into itself".

An elegant solution was given by Bratteli and Haagerup [1]. They showed that the conjecture was indeed true if $H$ and the modular operator $\Delta_{\xi}$ commuted and that this was necessary.

For the case of an inner limit derivation acting on a $C^{*}$-algebra $\mathfrak{A}$, we formulate a condition involving the approximating derivations and a separating state $\omega$ so that if the "core condition" is satisfied in the representation space of $\omega$, we obtain an automorphism group of the von Neumann algebra. The condition is vacuous in case $\omega$ is a trace and so we recover the Bratteli-Robinson result [3], with some additional information about the unitary group $t \rightarrow e^{i H t}$.

First we need an easy lemma about the skew information of an operator $h$ relative to a state $\omega$, which as indicated in [4] is "a measure of how far $h$ is from commuting with $\omega . "$

Lemma. Suppose that $\omega(x)=(x \xi \mid \xi)$ is a faithful state on a von Neumann algebra $\mathfrak{T}$. Let $J$ be the corresponding modular anti-unitary involution. On the selfadjoint portion of $\mathfrak{N}$ define the seminorms

$$
p(h)=\|(I-J) h \xi\|
$$

and

$$
q(h)=\sup _{\substack{x \in M \\ x \neq 0}}|\omega([h, x])| /\left(\omega\left(x^{*} x+x x^{*}\right)\right)^{1 / 2}
$$

then $1 / \sqrt{2} q(h) \leqslant p(h) \leqslant q(h)$.

Received by the editors December 28, 1978.

AMS (MOS) subject classifications (1970). Primary 46L10; Secondary 82A15.

${ }^{1}$ Partially supported by the National Science Foundation. 
Proof. As $\Re\left(\xi\right.$ is a core for $\Delta^{1 / 2},[9]$,

$$
p(h)=\|(I-J) h \xi\|=\sup \left|\left(\left(I-\Delta^{1 / 2}\right) h \xi \mid\left(I+\Delta^{1 / 2}\right) x^{*} \xi\right)\right|
$$

where the supremum runs over the dense set of vectors in the unit ball of the form $\left(I+\Delta^{1 / 2}\right) x^{*} \xi$. But

$$
\left(\left(I-\Delta^{1 / 2}\right) h \xi \mid\left(I+\Delta^{1 / 2}\right) x^{*} \xi\right)=-\omega([h, x])
$$

so that $p(h)=\sup \left\{|\omega([h, x])|:\left\|\left(I+\Delta^{1 / 2}\right) x^{*} \xi\right\| \leqslant 1\right\}$. Noting that

$$
\omega\left(x^{*} x+x x^{*}\right) \leqslant\left\|\left(I+\Delta^{1 / 2}\right) x^{*} \xi\right\|^{2} \leqslant 2 \omega\left(x^{*} x+x x^{*}\right)
$$

the proof of the lemma is complete.

Let $\mathfrak{U}$ be a $C^{*}$-algebra, $\delta$ a densely defined *-derivation on $\mathfrak{U}$ which is inner limit in the sense that there exists a sequence $h_{n}=h_{n}^{*} \in \mathfrak{A}$ such that $\delta(x)=\lim \left[i h_{n}, x\right]$ for all $x \in \mathscr{D}(\delta)$. (See [8] for $\mathfrak{A}$ hyperfinite and $\delta$ a normal *-derivation where this is always satisfied.) Suppose now that $\omega$ is a state and $\omega(\delta(x))=0$ for all $x \in \mathscr{D}(\delta)$. If $\left(\pi_{\omega}, \mathfrak{Q}_{\omega}, \xi\right)$ denotes the corresponding GNS triple then the definition

$$
i H \pi_{\omega}(x) \xi=\pi_{\omega}(\delta(x)) \xi, \quad x \in \mathscr{D}(\delta)
$$

gives a densely defined symmetric operator $H$ such that $\left[i H, \pi_{\omega}(x)\right] \eta=$ $\pi_{\omega}(\delta(x)) \eta$ for all $\eta \in \pi_{\omega}(\mathscr{D}(\delta)) \xi$. The state $\omega$ is said to be separating if $y \rightarrow(y \xi \mid \xi)$ is faithful on $\Re=\pi_{\omega}(\mathfrak{U})^{\prime \prime}$.

THEOREM. Let $\omega$ be a separating state on $\mathfrak{A}$. Let $\delta$ be an inner limit derivation with $\omega \circ \delta=0$, and approximating sequence $h_{n}$. Suppose that $\left|\omega\left(\left[h_{n}, x\right]\right)\right|^{2} \leqslant$ $K \omega\left(x^{*} x+x x^{*}\right)$. If $H$ is as above and is essentially selfadjoint on $\pi_{\omega}(\mathscr{D}(\delta)) \xi$, then $\operatorname{ad}\left(e^{i H t}\right)$ implements an automorphism group of $\mathfrak{N}$.

Proof. We show first that $(I-J) \pi_{\omega}\left(h_{n}\right) \xi$ converges weakly to zero. By the hypothesis and the above Lemma it follows that $(I-J) \pi_{\omega}\left(h_{n}\right) \xi$ has a subsequence $(I-J) \pi_{\omega}\left(h_{n_{j}}\right) \xi$ converging weakly to some vector $\eta \in \mathfrak{B}$. Let $x^{*} \in \mathfrak{R}$, such that $\left(I+\Delta^{1 / 2}\right) x^{*} \xi$ approximates $\eta$ (the set $\mathfrak{T} \xi$ is a core for $\Delta^{1 / 2}$ ). We can in fact choose $x$ and $j_{o}$ so that for $j \geqslant j_{o}$

$$
\left|\left((I-J) \pi_{\omega}\left(h_{n_{j}}\right) \xi \mid\left(I+\Delta^{1 / 2}\right) x^{*} \xi\right)\right| \geqslant 1 / 2
$$

but the calculation of the Lemma shows

$$
\left|\left((I-J) \pi_{\omega}\left(h_{n}\right) \xi \mid\left(I-\Delta^{1 / 2}\right) x^{*} \xi\right)\right|=\left|\omega\left(\left[h_{n}, x\right]\right)\right| .
$$

Thus for $j \geqslant j_{0},\left|\omega\left(\left[h_{n_{j}}, x\right]\right)\right| \geqslant 1 / 2$. The inequality $\left|\omega\left(\left[h_{n}, y\right]\right)\right|^{2} \leqslant K\left\{\omega\left(y^{*} y\right)+\right.$ $\left.\omega\left(y y^{*}\right)\right\}$ extends to all of $\mathscr{N}$ and allows us to replace $x$ by some $y \in \mathscr{D}(\delta)$ and conclude that

$$
\left|\omega\left(\left[h_{n_{j}}, y\right]\right)\right| \geqslant 1 / 4 \text { for } j \geqslant j_{0} .
$$

However, $\left[i h_{n}, y\right] \rightarrow \delta(y)$ and $\omega(\delta(y))=0$, a contradiction. Thus $\eta=0$. As this holds for all subsequences, $(I-J) \pi_{\omega}\left(h_{n}\right) \xi \rightarrow 0$, weakly.

Since $(I-J) \pi_{\omega}\left(h_{n}\right) \xi$ converges weakly to zero we may choose a sub- 
sequence, $(I-J) \pi_{\omega}\left(h_{n_{k}}\right) \xi$ which converges $C-1$ to zero in norm [10]. Relabeling and letting $T_{j}=1 / j \sum_{n=1}^{j} h_{n}$ we have $(I-J) \pi_{\omega}\left(T_{j}\right) \xi \rightarrow 0$ in norm as $j \rightarrow \infty$. It is a simple matter to verify that $\left[i T_{j}, x\right] \rightarrow \delta(x)$ as $j \rightarrow \infty$.

Define $H_{j}=\pi_{\omega}\left(T_{j}\right)-J \pi_{\omega}\left(T_{j}\right) J$. Then for $x \in \mathscr{D}(\delta)$ we have

$$
\begin{aligned}
H_{j} \pi_{\omega}(x) \xi & =\left[H_{j}, \pi_{\omega}(x)\right] \xi+\pi_{\omega}(x) H_{j} \xi \\
& =\left[\pi_{\omega}\left(T_{j}\right), \pi_{\omega}(x)\right] \xi+\pi_{\omega}(x)(I-J) \pi_{\omega}\left(T_{j}\right) \xi .
\end{aligned}
$$

The first term converges to $-i \pi_{\omega}(\delta(x)) \xi=H \pi_{\omega}(x) \xi$ and the second converges to zero by the above calculations. As $\pi_{\omega}(\mathscr{D}(\delta)) \xi$ is a core for $H$, it follows, [5, p. 428], $e^{i H_{j} t} \rightarrow e^{i H t}$ strongly. Since

$$
e^{i H_{j} t} y e^{-i H_{j} t}=e^{i \pi_{\omega}\left(T_{j}\right) t} y e^{-i \pi_{\omega}\left(T_{j}\right) t}
$$

for all $y \in \mathfrak{N}$, it follows that $e^{i H t} \cdot e^{-i H t}$ is an automorphism group of $\mathfrak{N}$.

REMARKS. 1. There is a slight difference between our core condition and that of [2] in that $\mathscr{D}(\delta)$ there is for the extension of $\delta$ to $\mathfrak{N}$.

2. It is straightforward to show that the bound in the skew information of $h_{n}$ is equivalent to

$$
\omega\left(e^{i h_{n} t} x e^{-i h_{n} t}\right) \leqslant e^{\gamma t} \omega(x)
$$

for $t \geqslant 0, x \geqslant 0$, and some constant $\gamma$. An inequality of the form

$$
\omega\left(e^{i h_{n} t} x e^{-i h_{n} t}\right) \leqslant f(t) \omega(x)
$$

is satisfied in some classical models [6]. Here $f(t)$ is a bounded continuous function on the real line.

3. One way to obtain the inequality on $h_{n}$, is related to the modular automorphism for $\omega$. In that case the constant $K$ could be given by the Bogoliubov inequality, [7], as $\sup _{n}\left|\left((\log \Delta) h_{n} \xi \mid h_{n} \xi\right)\right|$ provided this number is finite. This involves a certain amount of analyticity for the $h_{n}$ with respect to the modular automorphism. We note that if this automorphism group preserves $\mathfrak{A}$ and acts points norm continuously then the $h_{n}$ can be replaced by analytic elements.

For various conversations about this and related matters we are grateful to A. Connes, N. Hugenholtz and M. Takesaki.

\section{REFERENCES}

1. O. Bratteli and U. Haagerup, Unbounded derivations and invariant states, Comm. Math. Phys. 59 (1978), 79-95.

2. O. Bratteli and D. W. Robinson, Unbounded derivations of von Neumann algebras, Ann. Inst. H. Poincaré Sect. A (N.S.) 25 (1976), 139-164.

3. , Unbounded derivations and invariant trace states, Comm. Math. Phys. 46 (1976), 31-35.

4. A. Connes and E. Strrmer, Homogeneity of the state space of factors of type III, J. Functional Analysis 28 (1978), 187-196.

5. T. Kato, Perturbation theory for linear operators, Springer-Verlag, New York, 1966.

6. C. Marchioro, A. Pellegrinotti and M. Pulvirenti, Self-adjointness of the Liouville operator for infinite classical systems, Comm. Math. Phys. 58 (1978), 113-129. 
7. R. T. Powers, Resistance inequalities for KMS states of the isotropic Heisenberg model, Comm. Math. Phys. 51 (1976), 151-156.

8. R. T. Powers and S. Sakai, Unbounded derivations in operator algebras, J. Functional Analysis 19 (1975), 81-95.

9. M. Takesaki, Tomita's theory of modular Hilbert algebras and its applications, Lecture Notes in Math., vol. 128, Springer-Verlag, Berlin and New York, 1970.

10. S. Banach and S. Saks, Sur la convergence forte dans les champs $L^{p}$, Studia Math. 2 (1930), 51-57.

Department of Mathematics, Pennsylvania State University, University Park, PennSYLVANIA 16802 